\title{
Design of Reference Phantom for Quality Control of Conventional X-ray Radiography Units in Port Sudan
}

\author{
${ }^{1}$ Yousif M.Y. Abdallah, ${ }^{2}$ Wail H. Zaki, ${ }^{2}$ Babeker A.E. Ahmadoun, ${ }^{2}$ Abuobeada A. E. Musa \\ ${ }_{1}$ Department of Radiological Science and Medical Imaging, College of Applied Medical Science, Majmaah \\ University, Majmaah,11952, Saudi Arabia; \\ ${ }^{2}$ Applied Physics Department, Faculty of Applied Science, Red Sea University, Port Sudan, Sudan \\ y.yousif@mu.edu.sa
}

\begin{abstract}
The Quality Control (QC) system, based on simple, cheap equipment and minimum personnel time, enables a resource-limited facility and staff to control the fundamental components of the imaging process on a low cost basis. Quality Assurance (QA) is a product or service quality management program. Customer reviews, capacity building and quality control can also be included. Quality control requires specific measures for ensuring measurable process-related aspects of product output or for the delivery of services within a given limit. Research was conducted at the Medical Physics Department of Red Sea University. The main objective of this work was to boost quality assurance rays. The imagination is more user-friendly and produces better results than a person or object. Phantoms, including fluoroscopy or $x-$ rays, and certain image quality measurements have been used in $x$-rays imaging. The manufactured phantom in this study showed high precision in different QC tests.
\end{abstract}

Keywords: Reference, phantom, quality control, X-ray, Port Sudan.

\section{Introduction}

For internal organ screening and detection of abnormal body processes, medical diagnostic imaging is used. A physicians working in this area are radiologist with a radiology technologist who conducts several procedures for imaging [1], [2]. This relates to the first study of photographs that established the theory of radiology $(X)$ and radiology at the end of the 19th century. The Nobel Prize for short wave and highenergy electro-magnetic radiology was awarded in 1901 to develop the method. These rays come into the body, leaving with grapes a standard camera plate (like high)[4], [5]. For stain tissue to inject, redirect or intravenously into the bloodstream, contrasting materials (contrasting agents) are also required temporarily. It has many electrons, and the body is easily disposed of. However, Aunt William Rontgen can see the strength of these rays and their boundaries from the first shot of history [6]. The bone of the handcuff can be seen in the image, but no snapshot can discern other soft tissue details like muscles, tendons and blood vessels [7]. A QC system supports network and staff in monitoring various image process components with simple, cost-effective tools and time reduction at low costs [8]. The QA 
Framework is a management program that ensures the quality of the product or service. Other things can include a system such as customer feedback, employee satisfaction and quality control. Quality control includes specific actions for the management, within defined limits, of observable elements of product development or service delivery cycles [9]. In general, these tasks include measuring the variable rule, checking the value limit measured as well as corrective measures if the limit exceeds [10]. The study suggests variables such as measurement methods, control limits and certain corrections commonly used in radiological imagery to track the performance of equipment. Every medical facility using X-ray technology will benefit from the quality assurance programme, from a basic intraoral dental instrument through to a complex image-intensified procedures system [11]. An existing system tracks the imaging process from beginning to end and potentially shows unresolved problems. In the view of the Subcommittee, these standards and procedures form the basis for system management and may not meet the requirements of certain federal or state regulations. A rapidly growing discipline is quality assurance in medical imaging sponsored by unique facilities for continuous improvement of their programs [12][13]. Quality control guidelines were drawn up for radiographic or fluoroscopic devices. Wide varieties of dental and podiatric installations are available. Alternative structure provided by these complexing centers and an additional oxygen [14]. X-ray phantom is a special tool for storing, analyzing and recording, measuring and photographing the output of various imaging instruments in medicine. Imagination is easier to use and more effective than using a living person or a corpse, and prevents direct danger to a living person. First used in 2D x-rays imaging or fluoroscopy techniques and certain image quality measurements [15]. X-ray devices are complicated. Biomedical engineering staff needs a wealth of knowledge, service handbooks, and expensive testing and repair equipment. Simple test exposure with reliable and enough to check the tube performance. A simple test image shows whether the $\mathrm{x}$-ray tubes are sufficiently radiated and, if the collimator is properly calibrated, possible objects produced by the development process. One common problem is collimator mirror is incorrect [16], [17]. Therefore, the $x-$ ray projector and light beam are no longer concomitant, revealing a particular aspect in the radiograph. It includes a metal grid. The view in this metal grid shows how much and where the technician can shift the mirror. Constant testing requires an aluminum wedge. The aluminum bar slowly decreases [18] [19].

\section{Materials and Methods}

Specifically developed a phantom for assessing fluoroscopic image quality, properly absorbed and measured in either cycle $\mathrm{mm}$ or mesh, with low contrast levels in black and white contrast patches. Researchers removed the compression tool from the beam, positioning the region under clinical conditions (e.g. lighting, location of the grid, etc.). The center of the field was radiographed with using a test tool for enhancing the image, the attenuator and the quality test tool for beam. The radiographed field to the phantom device assessment tool was $10 \times 10 \mathrm{~cm}$. The phantom was put in a specific distance between the source and the image. Scientists monitor collimators properly by collimating the radiation fields to correspond to the edges of the phantom device. Fluoroscopic analysis of the proposed phantom's image quality showed the preservation of high and low contrast image quality devices as shown in figure 1. 

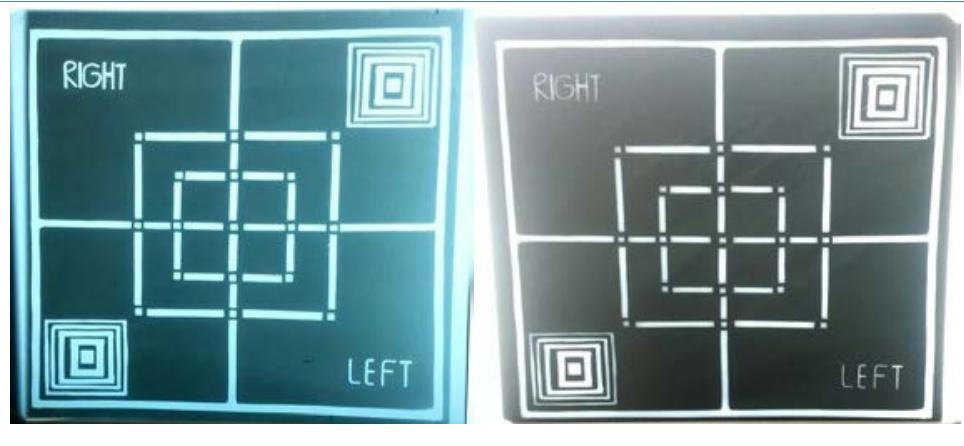

Figure 1. Shows radiographic image of x-rays phantom

\subsection{Steps of phantom manufacturing:}

- Step 1: Construction and Design of the phantom

- Step 2: Manufacture a metal model of the constructed phantom

- Step 3: The phantom case lead wires manufacturing

- Step 4: wedge manufacturing

- Step 5: Preparation of the wire pieces

- Step 6: Putting wire pieces into place

- Step 7: Calibration of the phantom

\section{The Results}

\subsection{Steps of phantom manufacturing:}

\subsubsection{Step 1: Construction and Design of the phantom}

Both transverse and coronal section of the phantom were drawn. The design plans of the phantom were printed out on translucent plastic cases as shown in figure 2.

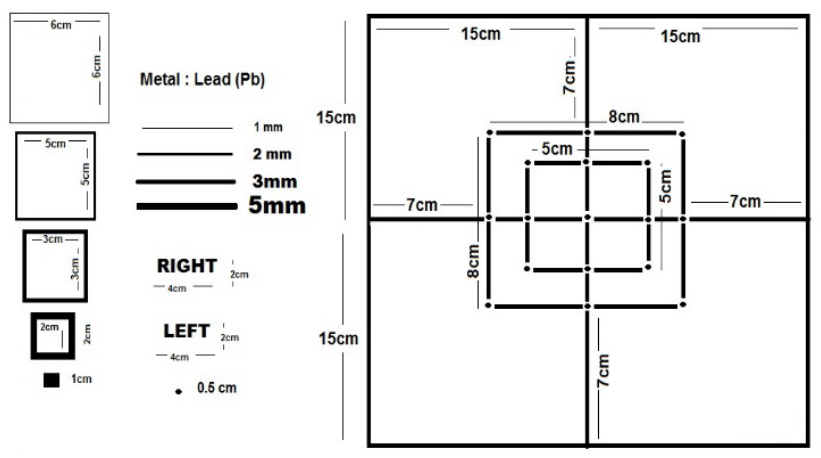




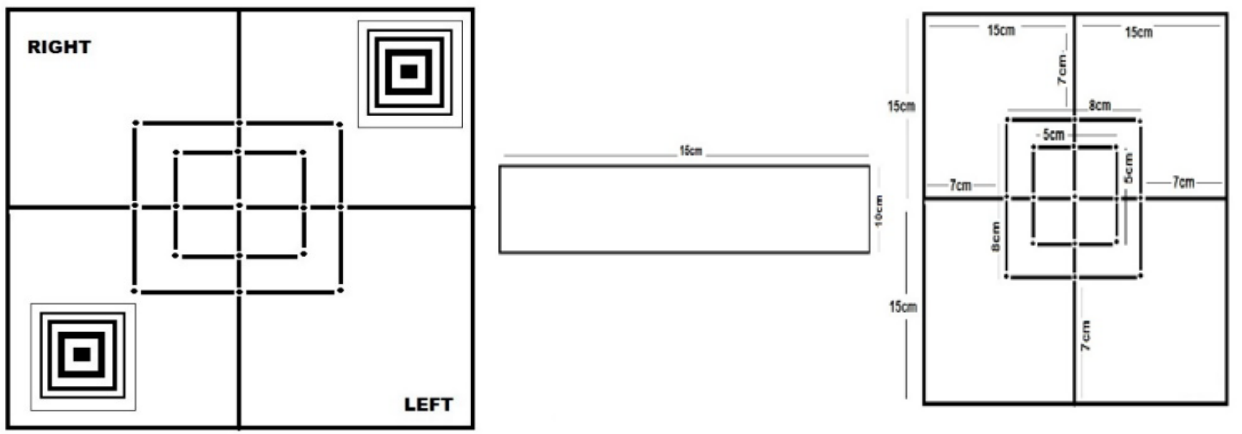

Figure 2. The manufactured phantom construction plans and layouts

\subsubsection{Step 2: Manufacture a metal model of the constructed phantom}

The phantom construction plans and layouts was placed on the plastic case as shown in figure 3.

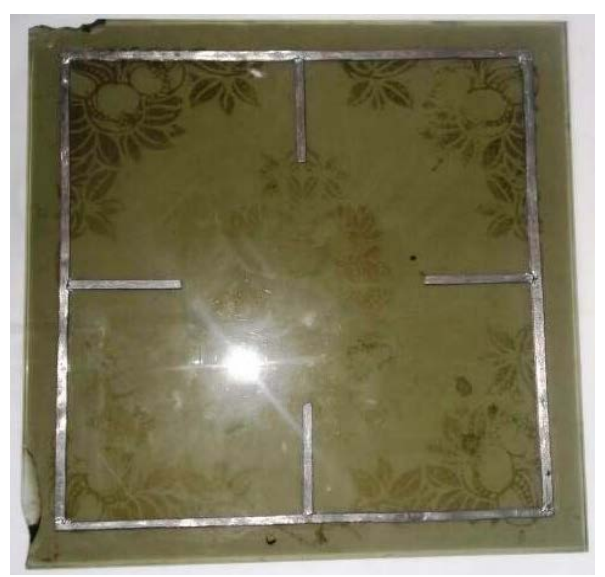

Figure 3. Phantom plastic case

\subsubsection{Step 3: The phantom case lead wires manufacturing}

Throughout the phantom plastic case, we fixed the lead wire - across for a rough overall and three squares of sizes and shapes at $5,8,15 \mathrm{~cm}$.

\subsubsection{Steps of lead wires melting and construction}

1. Lead materials for phantom as shown in figure 4.

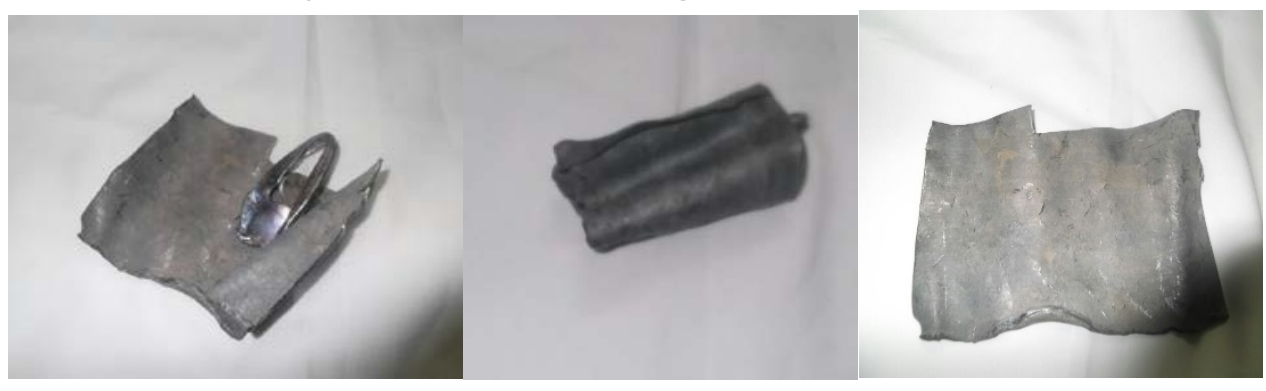

Figure 4. Lead sheet of manufactured phantom 
Yousif M.Y. Abdallah, Wail H. Zaki, Babeker A.E. Ahmadoun, Abuobeada A. E. Musa; Design of Reference Phantom for Quality Control of Conventional X-ray Radiography Units in Port Sudan. Journal of Biomedical Engineering and Medical Imaging, Volume 7, No 1, February (2020), pp 1-9

\section{2. $\quad$ Melting the lead wire as shown in figure 5.}

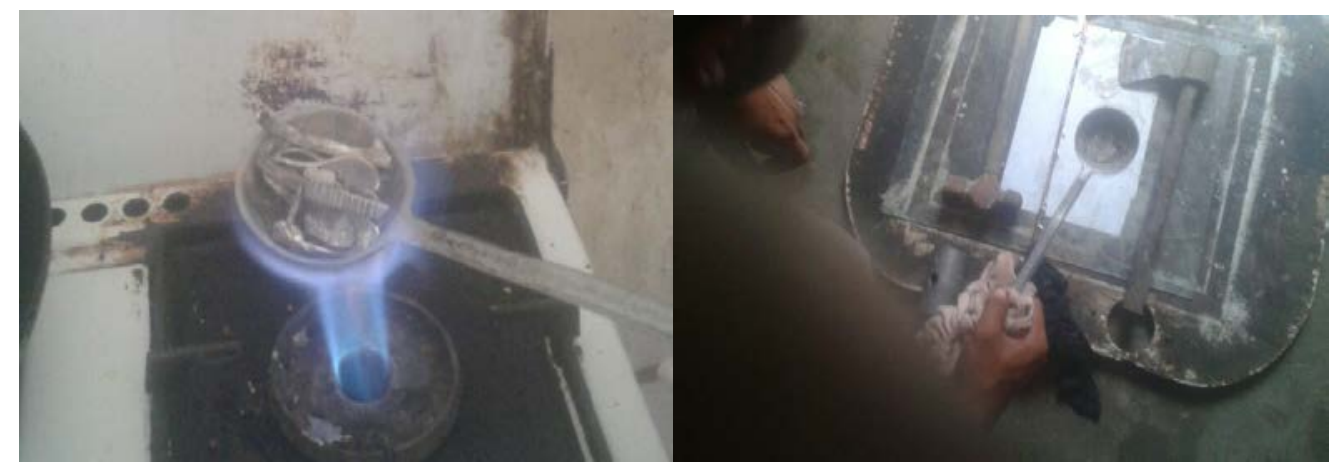

Figure 5. Melting process of lead

3. Trimming the lead sheet as shown in figure 6.
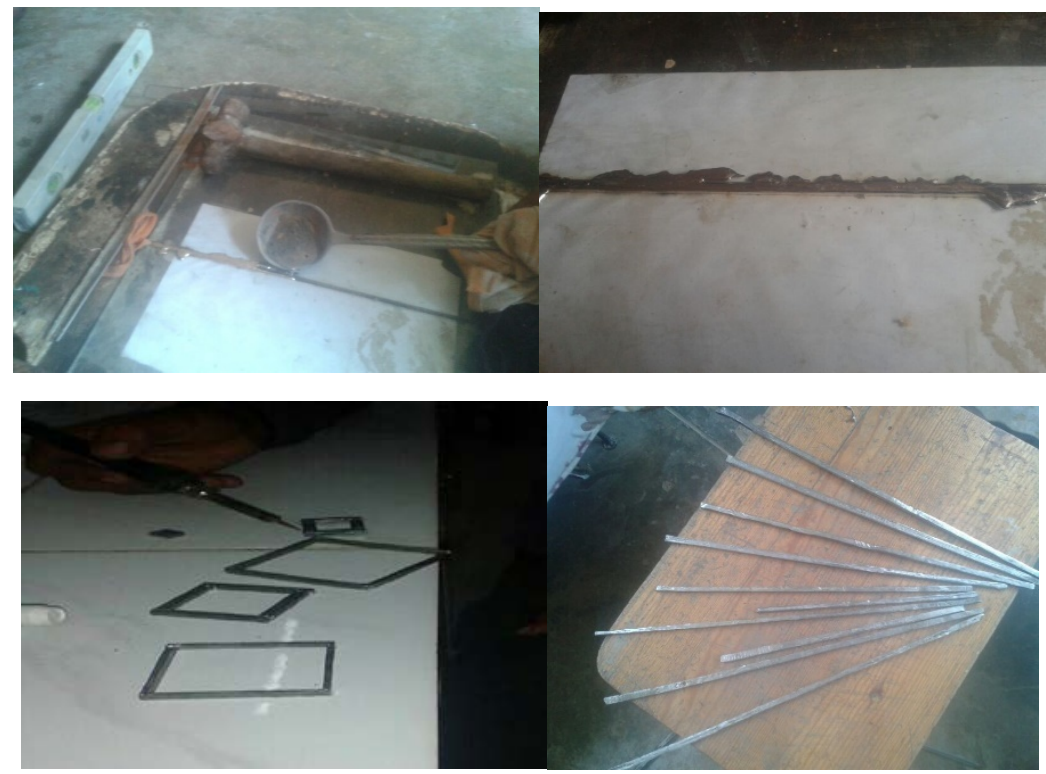

Figure 6. Show melding process of lead

4. Arrangement of the glued lead wires into three squares.

The glued lead wires were arranged into $5,8,15 \mathrm{~cm}$ squares. Later, this phantom would be radiological exposed concentrating on the central square as shown in figure 7 .
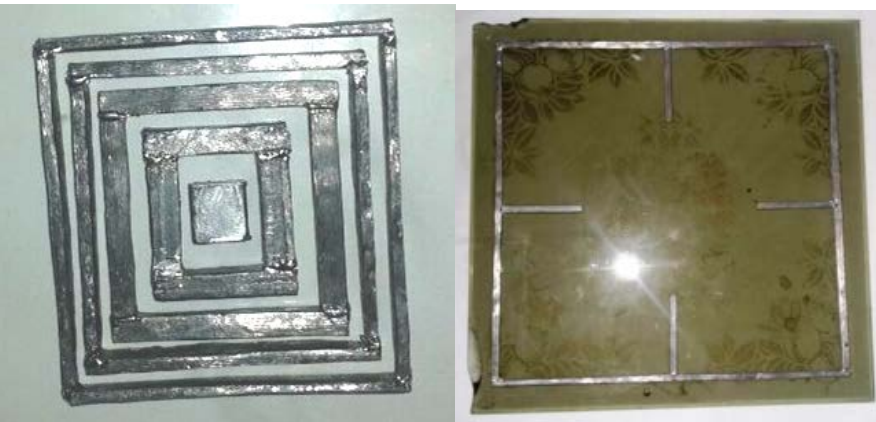

Figure 7. The arrangement of lead wires 


\subsubsection{Step 4: wedge manufacturing.}

We took old metal sheets and cut them into various sizes for the construction of the step wedge. With household shears, this was performed effectively. Trimming had always been simplified since it was needless to calculate and label. The strips with superglue were placed next to each other as shown in figure 8.

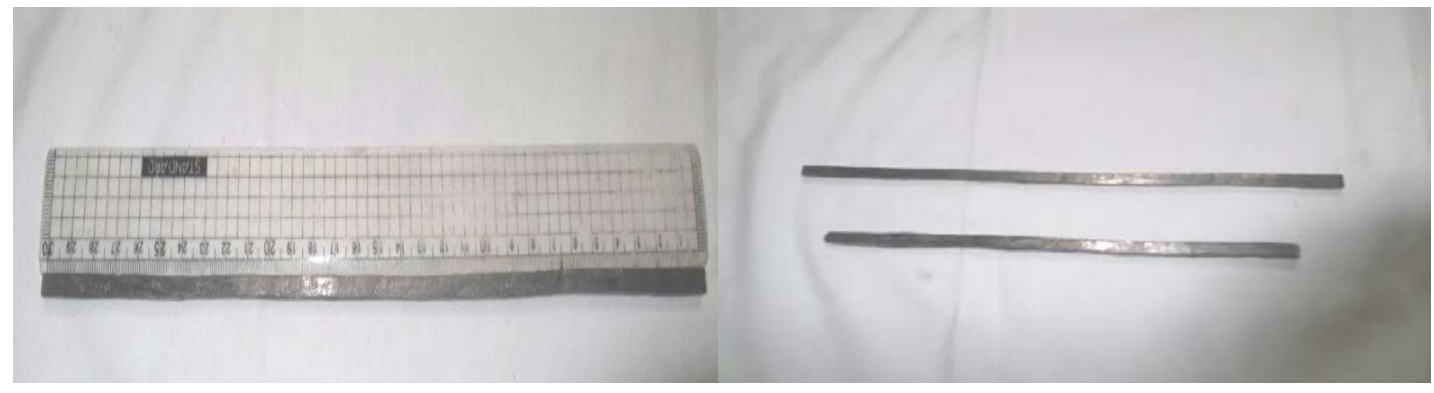

Figure 8. Lead wires

\subsubsection{Step 5: Preparation of the wire pieces.}

Half a meter of wire was taken and the separation eliminated. Instead, after locking one end of each wire into a table, we have stretched the cable, gripping the other end with a pair of pins and pushing quickly and securely. We bent the requisite wire parts and cut them. The bits in the corner were $5 \mathrm{~cm}$ long.

\subsubsection{Step 6: Putting wire pieces into place.}

We had printed the stencil plan and held it in the phantom box. We put the glue on the case and positioned the wire pieces in the desired positions as shown in figure 9.

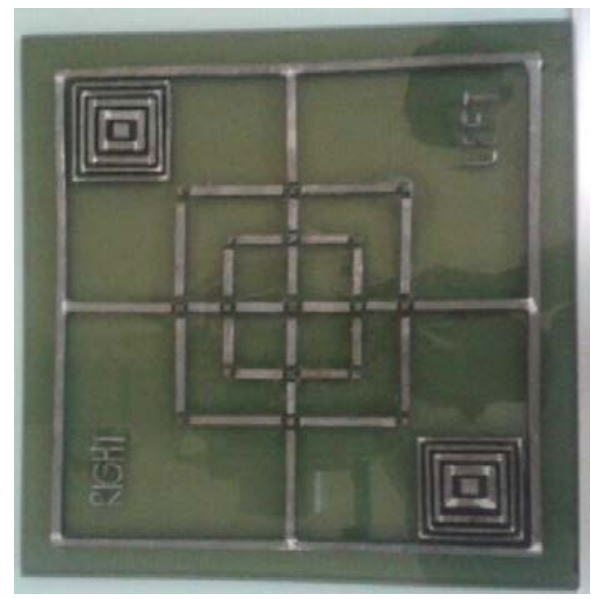

Figure 9. The final manufactured phantom

\subsubsection{Step 7: Calibration of the phantom:}

A powerful and well-maintained X-ray system was used to test the X-ray phantom. Exposures with different configurations had been made as shown in figure 10. 


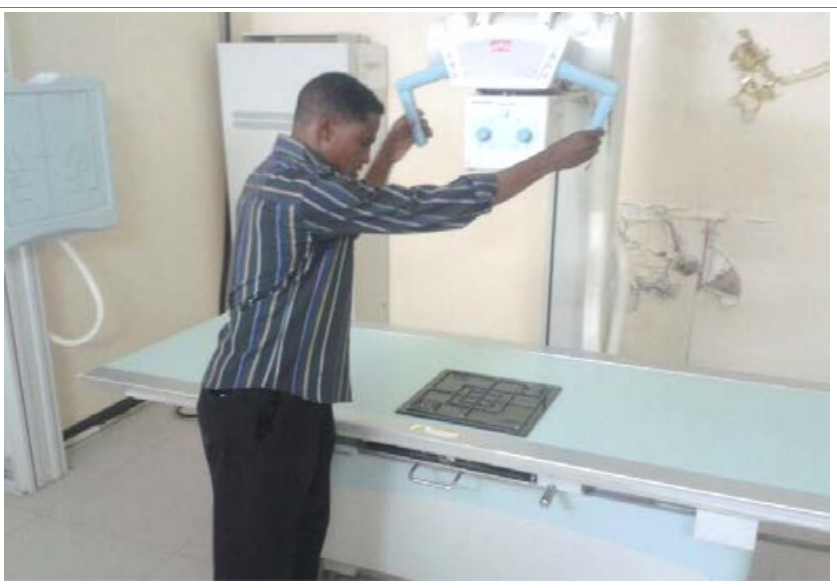

Figure 10. Testing the X-ray phantom

The grayscale is intended to be standardized. At $46 \mathrm{KV}-8 \mathrm{mAs}$ and $90 \mathrm{KV}-36 \mathrm{mAs}$, the phantom performs effectively as shown in figure 11 .

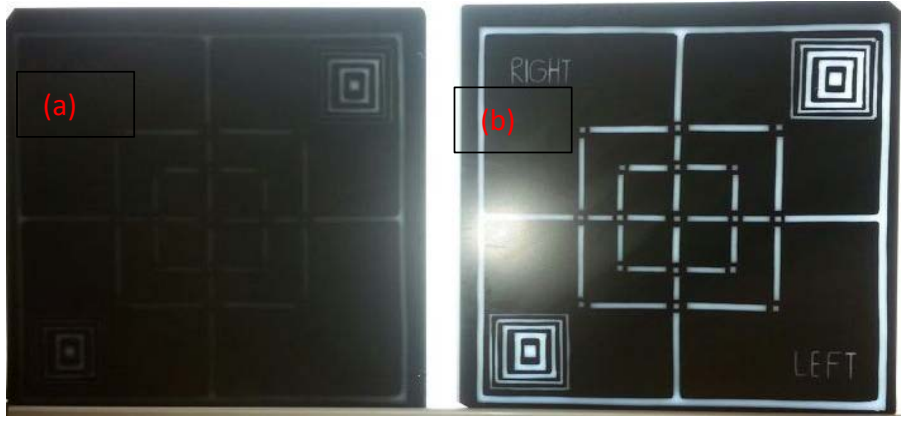

Figure 11. The results of phantom calibration image took at (a) $46 \mathrm{KV}-8 \mathrm{mAs}$, (b) $90 \mathrm{KV}-36 \mathrm{mAs}$

\section{Conclusion}

A phantom $x$-ray is an object designed to evaluate, analyze and tune the performance of diverse imaging instrumentation, scanned or imaged in the field of Medical Imaging. A phantom is a safer and more reliable than the use of a living human or cadaver, and protects a living being from being subject to direct risk. Phantoms were initially used in methods such as radiography or fluoroscopy for use with 2Dx-ray imaging and some image quality checking. Medical physicists need a wealth of knowledge, operating manuals and all the expensive test instruments for maintenance and repair. A quick, accurate x-ray phantom test exposure is appropriate. A simple test image shows whether the $\mathrm{x}$-ray tube produces adequate radiation and possible errors generated by the development process if the collimator has been calibrated properly. One common concern is that the collimator mirror is not correct. As a result, the $x-$ ray signal and the light beam are not consistent anymore and thus, the radiogram reveals a particular aspect. It is easy to use the proposed phantom. The phantom can be used to validate $x$-rays machines once the correct settings are set. The remainder of the photo should be shade-free. Exposure of the test phantom at the start of the day before the first patient is $\mathrm{X}$-radiated for the X-ray Technologist. Of multiple X-Ray, procedures for the $23 \mathrm{~cm}$ AP / LS spinal examination further validation tests were conducted to show the image clearly. The fabricated test phantom is easy to use and its results can be compared with the national median values. This interpretation could be used to render medical images compatible with 
the patient's radiation dose. For radiation detection, the $x$-ray QA Check phantom is available for digital and conventional imaging systems.

\section{REFERENCES}

[1]. Abdallah, Y., Hayder, A., and Wagiallah ,E., Automatic Enhancement of Mammogram using Contrast Algorithm. International Journal of Science and Research (IJSR), 2014. 3(9): p. 1886-1891.

[2]. Abdelwahab, R., and Abdallah, Y., Hayder, A., and Wagiallah ,E., Application of Texture Analysis Algorithm for Data Extraction in Dental X-Ray Images. International Journal of Science and Research (IJSR), 2014. 3(10): p. 1934-1939.

[3]. Abdallah, Y., Alkhir, M., Algaddal, A., Improvement of Brain Tumors Detection Using Markers and Boundaries Transform. International Journal of Science and Research (IJSR), 2015. 4(1): p. 2372-2378.

[4]. Bushberg, J. T., Seibert, J. A, Leidholdt, E. M. and Boone, J. M. The Essential Physics of Medical Imaging. 2nd ed. Lippincott William and Wilkins, Philadelphia. USA. 2003: p. 124-138

[5]. Vaño E., Miller D.L., Martin C.J., Rehani M.M., Kang K., Rosenstein M., Ortiz-Lopez P., Mattsson S., Padovani R., Rogers A., Diagnostic reference levels in medical imaging. ICRP Publication 135. Ann. ICRP 2017.46(1).

[6]. Abdallah, Y., Application of Analysis Approach in Noise Estimation, Using Image Processing Program. Lambert Publishing Press GmbH \& Co. KG, Germany, 2011.

[7]. Abdallah, Y., Introduction to Dental radiograpy, Theroy and pactice. Lambert Publishing Press GmbH \& Co. KG, Germany, 2019:102-123

[8]. Korner M, Weber CH, Wirth S, Pfeifer KJ, Reiser MF, Advances in Digital Radiography: Physical Principles and System Overview 1. Radiographics 2007.27(3): 675-686.

[9]. Lyra, M.; Sotiropoulos, M., Lagopati, N. \& Gavrilleli, M. , Quantification of Myocardial Perfusion in 3D SPECT images - Stress/Rest volume differences, Imaging Systems and Techniques (IST), IEEE International Conference 2010: p. $31-35$

[10]. Narasimha-lyer, H., et al., Automatic Identification of Retinal Arteries and Veins From Dual-Wavelength Images Using Structural and Functional Features. Biomedical Engineering, IEEE Transactions, 2007. 54(8): p. 1427-1435.

[11]. Abdallah, Y., and Wagiallah, E., Segmentation of Thyroid Scintography Using Edge Detection and Morphology Filters. International Journal of Science and Research (IJSR), 2014. 3(11): p. 2768-2772.

[12]. Abdallah YM, Boshara MA. Assessment of field size on radiotherapy machines using texture analysis. Sudan Med Monit 2014;9:5-10.

[13]. Hong, S., Optimal scheduling of tracing computations for real-time vascular landmark extraction from retinal fundus images. Information Technology in Biomedicine, IEEE Transactions on, 2001. 5(1): p. 77-91. 
Yousif M.Y. Abdallah, Wail H. Zaki, Babeker A.E. Ahmadoun, Abuobeada A. E. Musa; Design of Reference Phantom for Quality Control of Conventional X-ray Radiography Units in Port Sudan. Journal of Biomedical Engineering and Medical Imaging, Volume 7, No 1, February (2020), pp 1-9

[14]. Pinz, A., et al., Mapping the human retina. Medical Imaging, IEEE Transactions on, 1998. 17(4): p. 606-619.

[15]. Abdallah, Y., and Yousef R., Augmentation of X-Rays Images using Pixel Intensity Values Adjustments. International Journal of Science and Research (IJSR), 2015. 4(2): p. 2425-2430.

[16]. Wasan, B., et al., Vascular network changes in the retina with age and hypertension. Journal of hypertension, 1995. 13(12).

[17]. Koozekanani, D., et al., Tracking the Optic Nerve Head in OCT Video Using Dual Eigenspaces and an Adaptive Vascular Distribution Model. Computer Vision and Pattern Recognition, IEEE Computer Society Conference on, 2001. 1: p. 934. 